\title{
Identification of biomarkers with a tumor stage-dependent expression and exploration of the mechanism involved in laryngeal squamous cell carcinoma
}

\author{
LIAN HUI, NING YANG, HUIJUN YANG, XING GUO and XUEJUN JANG
}

Department of Otolaryngology, The First Hospital of China Medical University, Shenyang, Liaoning 110001, P.R. China

Received April 14, 2015; Accepted July 2, 2015

DOI: $10.3892 /$ or.2015.4230

\begin{abstract}
The aim of this study was to identify biomarkers with a tumor stage-dependent expression manner and explore the regulatory mechanisms of laryngeal squamous cell carcinoma (LSCC) progression. Microarray data GSE59102 was used for differential analysis using a limma package. Enrichment analyses were performed for the differentially expressed genes (DEGs) between tumor tissues and normal tissues at different stages. A co-expressed network involving the overlapped DEGs in two stages was established based on Pearson's correlation coefficients. Furthermore, for the tumor stage-dependent expressed DEGs, a protein-protein interaction (PPI) network was constructed by mapping the genes using the STRING database. Transcription factors (TFs), oncogenes and tumor-associated genes (TSGs) among the DEGs were predicted, following a search of the TRANSFAC, tumor-associated gene (TAG) and TSG databases. The CDT database was used to identify LSCC-associated genes. In total, 696 DEGs from early stage and control samples and 622 DEGs from advanced sttage and control samples were selected, which were mainly enriched in the cell cycle pathway. In the co-expressed network, BUB1, TTK, E2F1 and CEP55 were prominent, with E2F1 being predicted as a TSG and CEP55 as an oncogene. The HOX family members were predicted as TFs. MMP1, MMP9, MMP3 and PLAU were the most evident nodes in the PPI network, where MMP3 was connected with MMP1. The $A D H$ family was correlated with LSCC. Several biomarkers with tumor stage-dependent expression were identified including $M M P 1, M M P 3, M M P 9, P L A U$ and $A D H s$. Additionally, the dysregulated cell cycle pathway involving $B U B 1, T T K, E 2 F 1$ and CEP55, and the mediation of $M M P 1$ by $M M P 3$ as well as the predicted TF HOX, may all play significant roles in LSCC progression.
\end{abstract}

Correspondence to: Dr Lian Hui, Department of Otolaryngology, The First Hospital of China Medical University, No.155 Nanjing Bei Street, Shenyang, Liaoning 110001, P.R. China

E-mail: lian_hui1@163.com

Key words: laryngeal squamous cell carcinoma, co-expressed; overlapped, tumor stage-dependent expression, differentially expressed genes

\section{Introduction}

Head and neck squamous cell carcinomas (HNSCC), is the fifth most common type of cancer worldwide, with the most frequent type being laryngeal squamous cell carcinoma (LSCC) (1). As an aggressive neoplasm, LSCC is the second most predominant upper respiratory tract tumor and has a high mortality (2). In addition, it was reported that LSCC had a more frequent incidence among middle-aged and elderly males (3). Although advanced surgical treatments such as laryngectomy, radiotherapy and chemotherapy have been developed in the diagnosis and prevention of early LSCC, the only option for advanced LSCC has been radio-chemotherapy, which often did not achieve a positive clinical response, resulting in a poor prognosis of advanced LSCC $(4,5)$. The 5-year survival rate for LSCC was $64.2 \%$, as indicated in a meta-analysis study (6). Therefore, investigations have been conducted to identify new approaches such as biological therapeutic strategy, in order to provide more effective and accurate predictions and preventions of LSCC.

Previously, a cohort of gene markers was identified that was associated with LSCC, including matrix metalloproteinase 2 (MMP2), cluster of differentiation 24 (CD24) and U3 small nucleolar ribonucleoprotein protein (IMP3) (7-9). Notably, the mRNA expression of dopa decarboxylase (DDC) gene, which encodes the enzyme L-DOPA decarboxylase, was associated with tumor stage in HNSCC and thus this gene was proposed as a potential prognostic biomarker of LSCC (10). Furthermore, several genes were significantly distinct between early and late tumor stages of LSCC, including ERBB,$B C L 7 A$ and RECQL4 (11). A recent study included patients with LSCC in different tumor stages to clarify new biomarkers that had driven the progression of LSCC. Based on their finding that HOXC8 and $H O X D 11$ were essential for FADU cell expansion, the $H O X$ family members were suggested to be correlated with the development of LSCC (12). However, alterations of the specific genes expressed in the early and late stage of LSCC were rarely involved. Thus, we employed the microarray data GSE59102 and re-analyzed it, to identify crucial genes in both stages using differential analysis. Additionally, we emphasized the overlapped DEGs (differentially expressed genes) in the two stages and examined the potential associations between these DEGs using co-expression network and protein-protein interaction (PPI) network analyses. Transcription factors 
(TFs) and tumor-associated genes (TSGs) were also predicted following a comparison of the relevant databases. Using these bioinformatical methods, we aimed to identify novel gene markers exhibiting a tumor stage-dependent expression and explore the underlying regulatory mechanisms of LSCC progression.

\section{Materials and methods}

Microarray data. The expression profile with the accession number GSE59102 (12) was downloaded from the public Gene Expression Omnibus (GEO, http://www.ncbi.nlm.nih.gov/geo) database. The data set comprised 29 cancer and 13 margin samples (control) from patients undergoing surgical ablation of LSCC. The cancer samples were obtained from two different tumor stages: 14 in the early and 15 in the advanced stage. The platform for the gene expression calculation was Agilent014850 Whole Human Genome Microarray 4x44K G4112F (Agilent Technologies, Palo Alto, CA, USA) and the annotation files in this platform were also downloaded for the analysis in the present study.

Data preprocessing and differential analysis. Following normalization, the data were presented in the form of a gene expression matrix. Uniform distribution of the gene mean value in different samples was obtained and the probe value was mapped into the gene symbol based on the annotation files. If $\geq 1$ probe was mapped into one gene, the average probe value was calculated as the final expression value of this gene.

The differential analysis was performed between early and control samples and between advanced and control samples, respectively, recruiting the limma (Linear Models for Microarray Analysis) package of Bioconductor R (http://www. bioconductor.org/packages/release/bioc/html/limma.html) (13). The t-test method in limma was used to calculate the significance for the DEGs selection and the P-value was adjusted to FDR (false discover rate). The threshold were FDR $<0.01$ and $\mid \log 2$ (fold change) $\mid \geq 4$.

Functional enrichment analysis of the DEGs. The gene ontology (GO, http: //www. geneontology. org/) (14), biological process (BP) and Kyoto Encyclopedia of Genes and Genomes (KEGG, http://www.genome.jp/kegg/pathway.html) (15) pathway enrichment analyses were conducted for two sets of DEGs, respectively, using the Database for Annotation, Visualization and Integration Discovery (DAVID, http://david.abcc.Ncifcrf. gov/) database (16), which revealed the enriched GO terms and KEGG pathways based on the hypergeometric distribution. The cut-off value for significant terms was $\mathrm{P}<0.05$.

Identification of TFs and TSGs. The two sets of DEGs were mapped into the TRASFAC database (17) to screen the potential TFs among these DEGs. Additionally, following the combination with the information from the tumor-suppressor gene (TSG) database (18) and TAG (http://www.binfo.ncku. edu.tw/TAG/) database, all known oncogenes and TEGs of the DEGs were extracted.

Co-expressed analysis of DEGs identified in both early-and advanced-stage samples. Considering that there may be several genes that are important in the early- and advanced-stage samples and that there may be certain correlations between these genes, the two sets of identified DEGs were merged to establish a co-expressed network, using criteria of Pearson's correlation coefficients $>0.9$.

Association analysis between the DEGs and different tumor stages. The overlapped genes in the two sets of DEGs were screened out, and any up- or downregulated genes showing a tumor stage-dependent increasing (the gene expression in different samples had the following trend: advanced $>$ early $>$ control) or decreasing expression (the gene expression in different samples had the following trend: advanced $<$ early $<$ control) were selected.

Subsequently, a KEGG pathway enrichment analysis was carried out for the DEGs that were positively and negatively associated with the tumor stage. As proteins often co-operate to exert their biological functions, examination of the PPI between them significantly contributes to identifying the underlying molecular mechanisms. Therefore, the above DEGs were mapped into the STRING (Search Tool for the Retrieval of Interacting Genes) (http://string-db.org/) database (19), to search the potential relationships between them from the protein level. The pairwise interactions of the DEGs (the protein products) with a combined score of $>0.4$ were selected to establish the PPI network, as visualized by the Cytoscape software (http://cytoscape.org/) (20).

Prediction of other disease-related genes. To examine the association between LSCC stage and DEGs, the screened overlapped tumor stage-dependent DEGs were mapped into the Comparative Toxicogenomics Database (CTD, http:// ctdbase.org/) (21), which collected disease-related genes that were validated by experiment or reported in the literature, to search for the exact LSCC-associated DEGs.

\section{Results}

Identification of DEGs in different stages. Based on the aforementioned selection criteria, a set of 258 upregulated and 438 downregulated DEGs between early-stage and control samples, and a cohort of 294 upregulated and 328 downregulated DEGs between advanced-stage and control samples were identified. The heat map of the cluster analysis of the DEGs is shown in Fig. 1.

Dysregulated biological functions and pathways of the DEGs. To characterize the biological functions altered by the DEGs, the GO functional and KEGG pathway enrichment analyses were carried out. As shown in Table I, of the DEGs between early-stage and control samples, the upregulated genes were significantly enriched in the cell cycle-related process such as M phase (GO: 0000279), cell cycle phase (GO: 0022403), cell cycle (GO: 0007049) and cell cycle process (GO: 0022402) and pathways including cell cycle (Hsa04110), cytokine-cytokine receptor interaction (Hsa04060) and homologous recombination (Hsa03440), while the over-represented BP terms and pathways for the downregulated genes were chemical homeostasis (GO:0048878), cation homeostasis (GO:0055080), response to wounding (GO:0009611), 
Table I. Significantly enriched biological process and pathways among the DEGs in the early LSCC samples (top five ranked by the P-value).

\begin{tabular}{lcc}
\hline Term & Count & P-value \\
\hline Upregulated DEGs & & \\
GO:0000279 M phase & 28 & $1.44 \mathrm{E}-14$ \\
GO:0022403 cell cycle phase & 30 & $8.34 \mathrm{E}-14$ \\
GO:0007049 cell cycle & 40 & $1.34 \mathrm{E}-13$ \\
GO:0022402 cell cycle process & 33 & $1.28 \mathrm{E}-12$ \\
GO:0001501 skeletal system development & 24 & $2.19 \mathrm{E}-11$ \\
Hsa04110: Cell cycle & 10 & $1.27 \mathrm{E}-06$ \\
Hsa04060: Cytokine-cytokine receptor interaction & 12 & $4.24 \mathrm{E}-05$ \\
Hsa05323: Rheumatoid arthritis & 7 & $7.67 \mathrm{E}-05$ \\
Hsa05146: Amoebiasis & 7 & $2.01 \mathrm{E}-04$ \\
Hsa03440: Homologous recombination & 4 & $2.73 \mathrm{E}-04$ \\
Downregulated DEGs & & \\
GO:0048878 chemical homeostasis & 27 & $1.58 \mathrm{E}-05$ \\
GO:0055080 cation homeostasis & 18 & $7.47 \mathrm{E}-05$ \\
GO:0009611 response to wounding & 26 & $7.59 \mathrm{E}-05$ \\
GO:0006957 complement activation, alternative pathway & 5 & $1.88 \mathrm{E}-04$ \\
GO:0030003 cellular cation homeostasis & 16 & $2.12 \mathrm{E}-04$ \\
Hsa04970: Salivary secretion & 11 & $2.75 \mathrm{E}-06$ \\
Hsa00982: Drug metabolism - cytochrome P450 & 10 & $3.09 \mathrm{E}-06$ \\
Hsa00980: Metabolism of xenobiotics by cytochrome P450 & 9 & $1.88 \mathrm{E}-05$ \\
Hsa00830: Retinol metabolism & 7 & $4.20 \mathrm{E}-04$ \\
Hsa04610: Complement and coagulation cascades & 7 & $6.67 \mathrm{E}-04$
\end{tabular}

DEGs, differentially expressed genes; LSCC, laryngeal squamous cell carcinoma; GO, gene ontology; Count, the DEG numbers enriched in a specific process or a pathway.
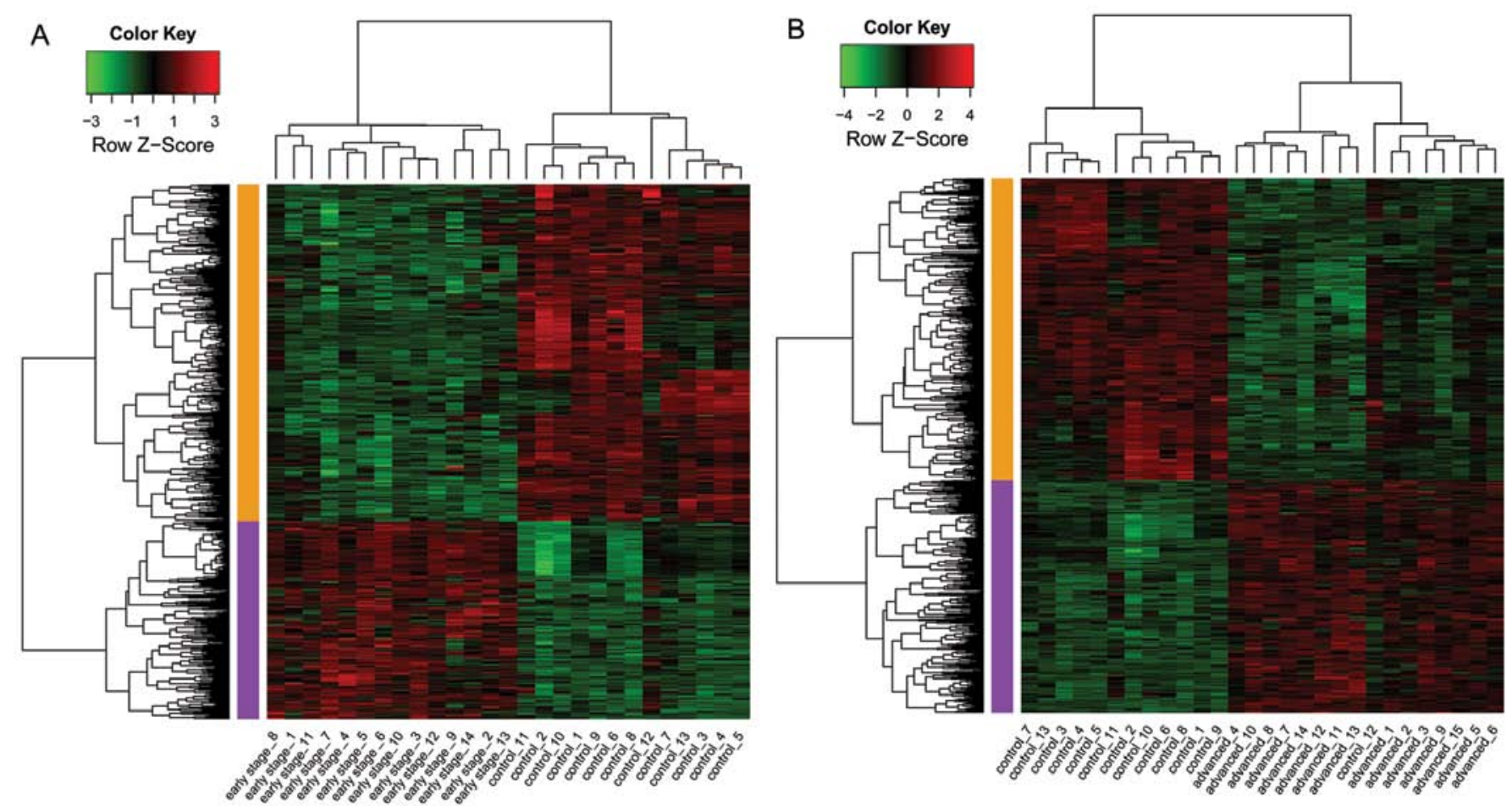

Figure 1. Heat map of the cluster analysis of the DEGs in LSCC. The X-axis shows the samples, while the Y-axis shows the genes. (A) Cluster analysis of DEGs between early-stage and control samples, and (B) advanced-stage and control samples. DEGs, differentially expressed genes; LSCC, laryngeal squamous cell carcinoma. 
Table II. Significantly enriched biological process and pathways among the DEGs in advanced-stage LSCC (top five ranked by the P-value).

\begin{tabular}{lcc}
\hline Term & Count & P-value \\
\hline Upregulated DEGs & 26 & $4.33 \mathrm{E}-16$ \\
GO:0007398 ectoderm development & 25 & $8.42 \mathrm{E}-16$ \\
GO:0008544 epidermis development & 25 & $1.41 \mathrm{E}-10$ \\
GO:0001501 skeletal system development & 12 & $4.70 \mathrm{E}-09$ \\
GO:0030216 keratinocyte differentiation & 12 & $1.22 \mathrm{E}-08$ \\
GO:0009913 epidermal cell differentiation & 19 & $4.26 \mathrm{E}-10$ \\
Hsa04060: Cytokine-cytokine receptor interaction & 8 & $5.38 \mathrm{E}-05$ \\
Hsa05146: Amoebiasis & 7 & $9.33 \mathrm{E}-05$ \\
Hsa04512: ECM-receptor interaction & 10 & 0.000127 \\
Hsa04062: Chemokine signaling pathway & 7 & 0.000144 \\
Hsa05323: Rheumatoid arthritis & & \\
Downregulated DEGs & 26 & $1.55 \mathrm{E}-04$ \\
GO:0055114 oxidation reduction & 13 & $2.01 \mathrm{E}-04$ \\
GO:0006631 fatty acid metabolic process & 7 & $9.67 \mathrm{E}-04$ \\
GO:0048754 branching morphogenesis of a tube & 14 & 0.001146 \\
GO:0007626 locomotory behavior & 7 & 0.001536 \\
GO:0050678 regulation of epithelial cell proliferation & 11 & $3.32 \mathrm{E}-07$ \\
Hsa04970: Salivary secretion & 10 & $4.42 \mathrm{E}-07$ \\
Hsa00982: Drug metabolism - cytochrome P450 & 9 & $3.35 \mathrm{E}-06$ \\
Hsa00980: Metabolism of xenobiotics by cytochrome P450 & 8 & $1.34 \mathrm{E}-05$ \\
Hsa00830: Retinol metabolism & 7 & $6.74 \mathrm{E}-05$ \\
Hsa00590: Arachidonic acid metabolism & &
\end{tabular}

DEGs, differentially expressed genes; LSCC, laryngeal squamous cell carcinoma; GO, gene ontology; Count, the DEG numbers enriched in a specific process or a pathway.

cellular cation homeostasis (GO:0030003), salivary secretion (Hsa04970), drug metabolism - cytochrome P450 (Hsa00982), metabolism of xenobiotics by cytochrome P450 (Hsa00980) and retinol metabolism (Hsa00830).

The result of the functional enrichment analysis of the DEGs between the advanced-stage and control samples is presented in Table II. The upregulated genes were predominantly enriched in BP terms including ectoderm development (GO: 0007398), epidermis development (GO: 0008544), skeletal system development (GO: 0001501) and epidermal cell differentiation (GO: 0009913), as well as the pathways involved in cytokine-cytokine receptor interaction (Hsa04060), ECM-receptor interaction (Hsa04512) and chemokine signaling pathway (Hsa04062). For the downregulated genes, the notable BP terms were oxidation reduction (GO: 0055114), fatty acid metabolic process (GO: 0006631) and regulation of epithelial cell proliferation (GO: 0050678), while the predominant pathways included were drug metabolism-cytochrome P450 (Hsa00982), metabolism of xenobiotics by cytochrome $\mathrm{P} 450$ (Hsa00980) and retinol metabolism (Hsa00830).

TFs, oncogenes and TSGs identification among the DEGs. Based on the information in the relevant databases, the potential TFs, oncogenes and TSG among the DEGs were screened out. As shown in Table III, a set of upregulated HOX family members as well as the downregulated $S M A D 9$, $P P A R G, L M O 3, N K X 3-1$ and $K L F 15$ were identified as the TFs. The upregulated HMGA2, HOXA10, LAMC2, CEP55 (centrosomal protein $55 \mathrm{kDa}$ ) as well as $\mathrm{LMO2}, \mathrm{CD} 24$ and $L C N 2$ were predicted as the oncogenes. Additionally, the upregulated genes including MMP11,E2F1 and BRCA2 as well as the downregulated MUCI, SERPINI2 and CEACAM1 were identified as TSGs, amongst the DEGs in the early-stage samples.

By contrast, in the advanced-stage samples, a number of upregulated HOX family members were identified as TFs, together with the downregulated DEGs such as NKX6-2,ELF5 and $N K X 3-1$. The potential oncogenes were the upregulated LAMC2, HOXA10 and CEP55 as well as the downregulated $L C N 2$ and $W I S P 2$. The upregulated $M M P 11, E 2 F 1$ and $D E C 1$ as well as the downregulated DEGs including DEFB1, MUC1 and ACCN1 were deemed as the TSGs.

Co-expressed network of all the DEGs and the functional enrichment analysis. According to the Pearson's correlation coefficients, the co-expressed network was constructed, comprising 494 pairwise interactions and 205 DEGs (Fig. 2). A gene in the network served as a 'node' and the 'degree' of a node referred to the interaction number of that specific gene. 
Table III. The TFs, oncogenes and TSGs among the DEGs.

\begin{tabular}{|c|c|c|c|}
\hline DEGs & $\mathrm{TF}$ & Oncogene & TSG \\
\hline \multicolumn{4}{|c|}{ DEGs in early-stage samples } \\
\hline Upregulated genes & 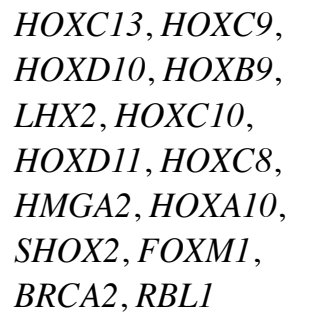 & $\begin{array}{l}\text { HMGA2, HOXA10, } \\
L A M C 2, C E P 55\end{array}$ & $\begin{array}{l}\text { IL24, ISG15, MMP11, TMEFF1, } \\
\text { CHEK1, E2F1, BRCA2, } \\
\text { MFHAS1, RBL1 }\end{array}$ \\
\hline Downregulated genes & $\begin{array}{l}\text { SMAD } 9, P P A R G, \\
L M O 3, N K X 3-1, \\
\text { KLF15, SOX10, } \\
\text { EHF, TFAP2B, } \\
\text { FOXA1, NR3C2, } \\
\text { MEOX2, AR }\end{array}$ & $\begin{array}{l}L M O 2, C D 24, \\
L C N 2, W I S P 2\end{array}$ & $\begin{array}{l}\text { MUC1, SERPINI2, CEACAM1, } \\
\text { GPX3, PEG3, SYT13, } \\
\text { EHF, ZBTB16, SLC5A8, } \\
\text { RARRES1, NDRG2, PPP1R3C, } \\
\text { CEACAM7, SFRP1, CLU, } \\
\text { LGI1, SRPX, PPP1R1B, } \\
\text { BAI3, HPGD, MAL, } \\
\text { LTF }, P L A 2 G 2 A, C 2 \text { orf } 40, \\
\text { MSMB, DMBT1, SCGB3A1 }\end{array}$ \\
\hline \multicolumn{4}{|c|}{ DEGs in advanced-stage samples } \\
\hline Upregulated genes & 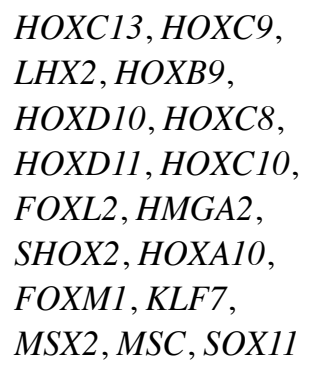 & $\begin{array}{l}\text { LAMC2, HMGA2, } \\
\text { HOXA10, WISP1, } \\
\text { CEP } 55, \text { SALLA, } \\
\text { CDC } 25 B, \text { MLF1, } \\
\text { FOSL1 }\end{array}$ & $\begin{array}{l}\text { IL24, ISG15, } \\
\text { MMP11,TMEFF1, } \\
\text { E2F1, AIM2, } \\
\text { SOX11, DEC1 }\end{array}$ \\
\hline Downregulated genes & $\begin{array}{l}\text { NKX6-2, ELF5, } \\
\text { NKX3-1,EHF, } \\
\text { HLF, PAX9, } \\
\text { MEOX2, NR3C2, } \\
\text { AR, TFAP2B, } \\
\text { FOXA1 }\end{array}$ & $L C N 2, W I S P 2$ & $\begin{array}{l}\text { DEFB1, MUC1, } \\
\text { ACCN1,EHF, } \\
\text { PEG3,CLU, } \\
\text { CEACAM1, SLIT2, } \\
\text { NDRG } 2, \text { SRPX, } \\
\text { LGI1,CEACAM7, } \\
\text { GPX3, HPGD, } \\
\text { PPP1R3C, PPP1R1B, } \\
\text { BAI3, MSMB, } \\
\text { PLA2G2A,C2orf40, } \\
\text { MAL,DMBT1, } \\
\text { SCGB3A1 }\end{array}$ \\
\hline
\end{tabular}

TF, transcription factor; TSG, tumor-associated genes; DEG, differentially expressed genes.

In the network, the nodes with high degrees were prominent such as BUB1 (degree =35), BIRC5 (degree =31), TPX2 (degree $=28)$, TTK (degree $=25)$, FAM72D (degree $=25$ ), CEP55 (degree $=24)$, CDC45 (degree $=23$ ), CDC25C (degree $=23$ ), E2F1 (degree =22) and DTL (degree =22).

The functional enrichment analysis revealed that, the $B U B 1, T T K$ and $C D C 45$ genes in the network were mainly enriched in the cell cycle pathway, whereas BIRC5 was associated with the cancer pathway. Notably, $E 2 F 1$ was involved in the two pathways.
PPI network of the tumor stage-dependent expressed DEGs and the enrichment analysis. Among the overlapped DEGs identified in the two samples, a set of 113 upregulated genes indicated an increasing expression in a tumor stage-dependent manner, while 115 downregulated genes exhibited a tumor stage-dependent decreasing expression. Combining the information from the STRING database, the PPI network of the tumor stage-dependent DEGs was constructed, comprising 125 interactions and 88 nodes (Fig. 3). As shown in Fig. 3, the highlighted nodes with high degrees were IL1B (degree $=13$ ), 


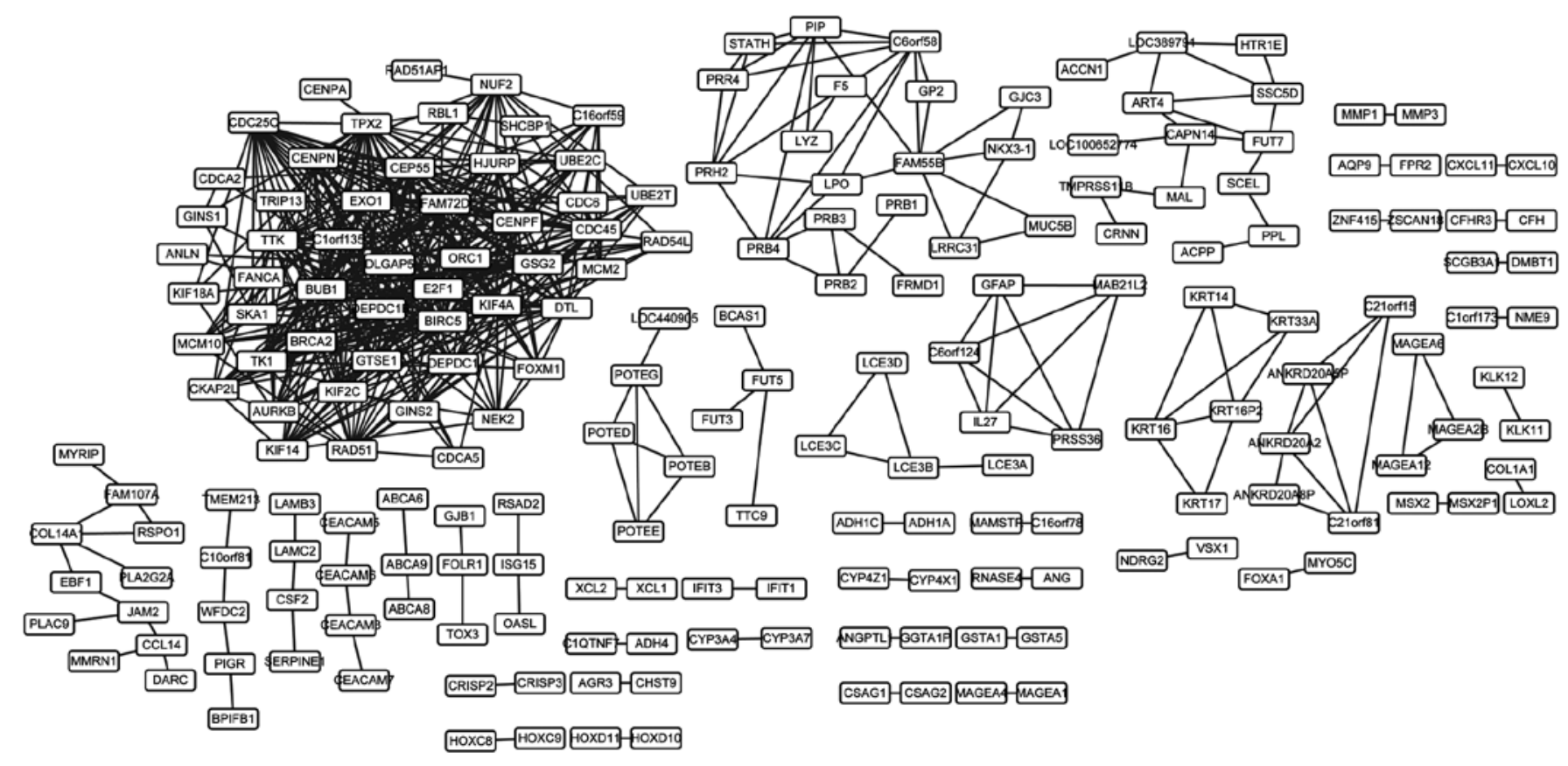

Figure 2. Co-expressed network of the overlapped DEGs in two stage samples of LSCC. Line in dash indicates the correlations between genes. DEGs, differentially expressed genes; LSCC, laryngeal squamous cell carcinoma.

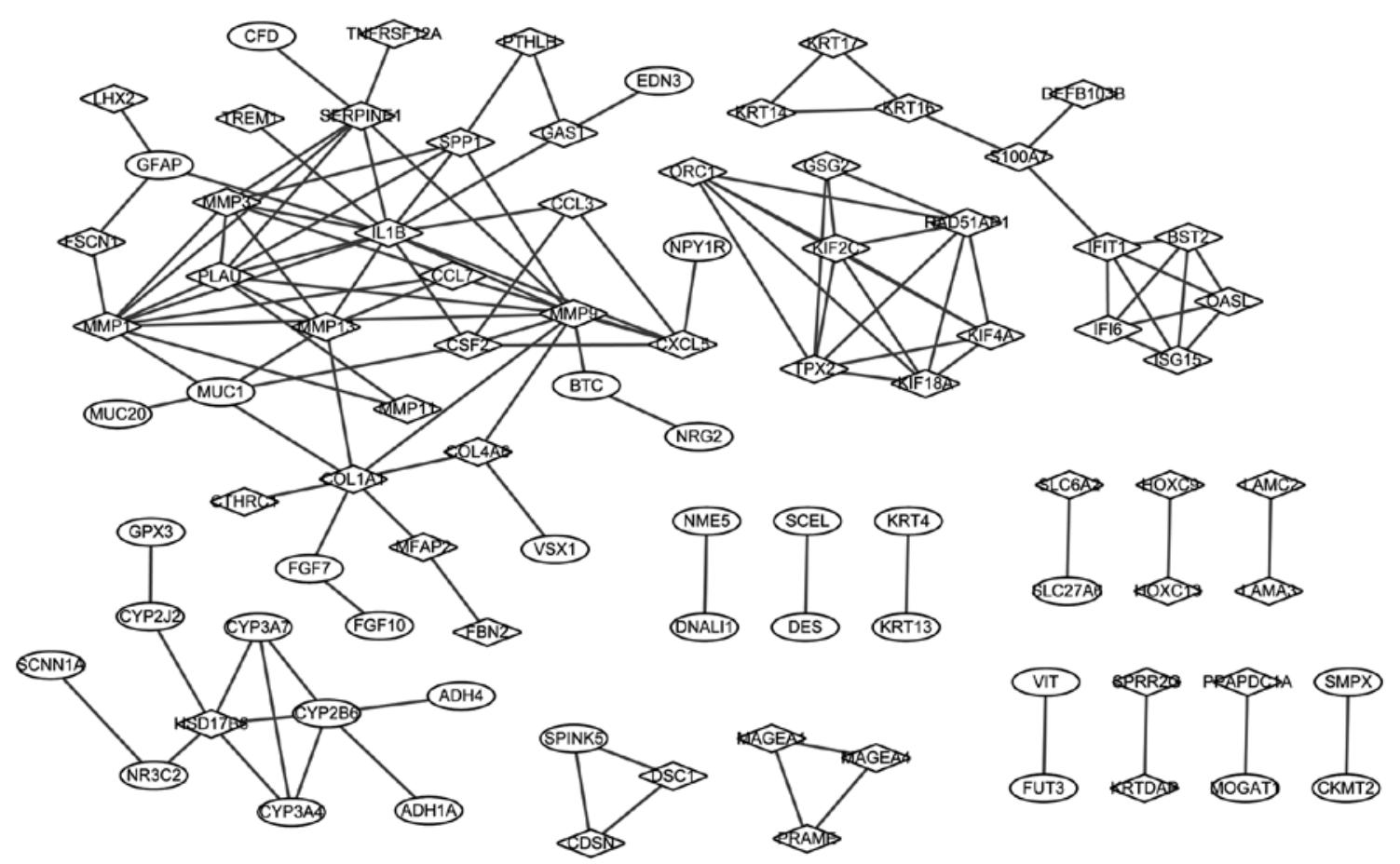

Figure 3. PPI network of the tumor stage-dependent expressed DEGs in two stage samples of LSCC. Red square denotes the upregulated DEG and green square denotes the downregulated DEG. Undirected line represents the interaction between genes. DEGs, differentially expressed genes; LSCC, laryngeal squamous cell carcinoma; PPI, protein-protein interaction.

MMP9 (degree =10), MMP1 (degree =9), PLAU (degree =8), SERPINE1 (degree $=7$ ), MMP3 (degree $=7$ ) and COL1A1 (degree $=7$ ).

LSCC-related genes among the tumor stage-dependent genes. By combining the CTD database and the tumor stage-dependent genes, 65 upregulated and 67 downregulated
DEGs were detected to be closely associated with LSCC via the curated literature. Notably, the $A D H$ family members of $A D H 7$ and $A D H 1 B$ were considered as two markers of LSCC. The expression of ADH family members including $A D H 1 A$, $A D H 1 C, A D H 4$ and $A D H 7$ during different stages is shown in Fig. 4.Analysis of variance (ANOVA) of the expression of four genes among different samples are presented in 
Table IV. False discovery rate of four gene expressions among different samples.

\begin{tabular}{|c|c|c|c|c|}
\hline Samples & $A D H 1 A$ & $A D H 1 C$ & ADH4 & $A D H 7$ \\
\hline Early stage and control & $1.05 \mathrm{E}-02$ & $2.41 \mathrm{E}-02$ & $4.00 \mathrm{E}-04$ & 2.64E-02 \\
\hline Advanced stage and control & $1.11 \mathrm{E}-03$ & $3.77 \mathrm{E}-03$ & $1.06 \mathrm{E}-04$ & $2.11 \mathrm{E}-03$ \\
\hline Advanced stage and beginning & $9.48 \mathrm{E}-01$ & $9.52 \mathrm{E}-01$ & $8.50 \mathrm{E}-01$ & $7.58 \mathrm{E}-01$ \\
\hline Early stage, advanced stage and control & $3.87 \mathrm{E}-03$ & $1.22 \mathrm{E}-02$ & $1.99 \mathrm{E}-06$ & $6.11 \mathrm{E}-03$ \\
\hline
\end{tabular}

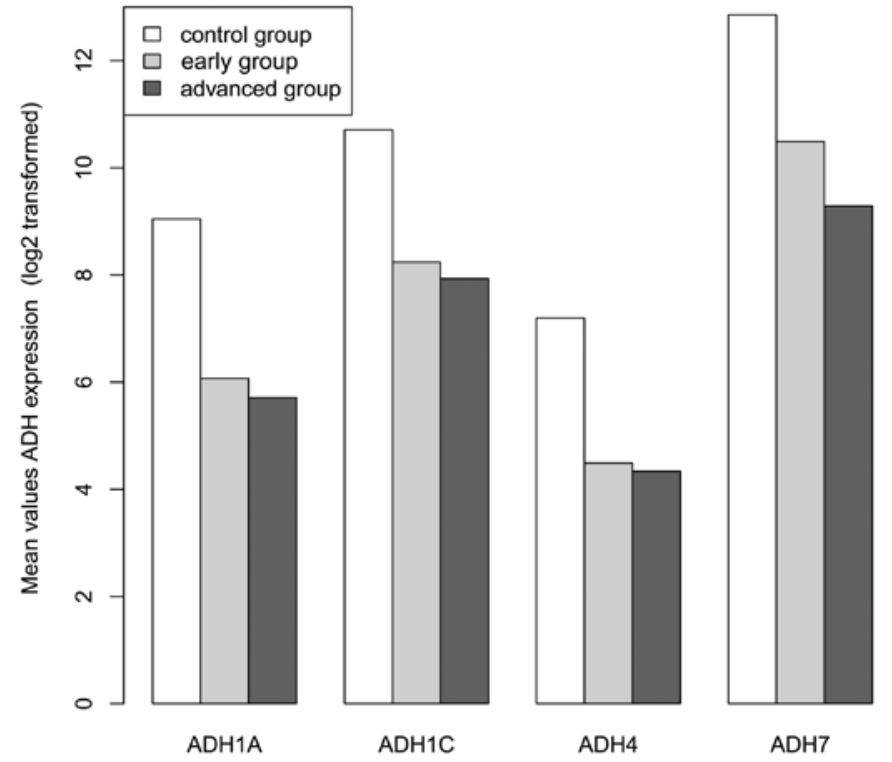

Figure 4. The expression of four ADH genes in contol, early and advanced stages of LSCC. LSCC, laryngeal squamous cell carcinoma.

Table IV, which indicated pronounced expression differences $($ FDR $<0.05)$ among these samples.

\section{Discussion}

In the present study, we identified 696 DEGs (258 upregulated and 438 downregulated) between early-stage and control samples, which were enriched in the cell cycle-related pathways, as well as 622 DEGs (294 upregulated and 328 downregulated) between advanced-stage and control samples with the over-represented pathways including the cytokine-cytokine receptor interaction and $\mathrm{ECM}$-receptor interaction pathways. In the co-expressed network, BUB1, TTK and E2F1, which were also predicted as potential TSGs, were prominent and mainly enriched in the cell cycle pathway. CEP55, also evident in the co-expressed network, was identified as an oncogene. The HOX family members were predicted as TFs in the two samples. The upregulated MMP1, MMP9 and MMP3 were the predominant nodes in the PPI network and had a tumor stage-dependent increasing expression pattern. Additionally, MMP3 was connected with MMP1 in the PPI network. $A D H$ family ( $A D H 1 A, A D H 1 C, A D H 4$ and $A D H 7)$ were predicted to be correlated with LSCC.

The gene $B U B 1$ encodes a serine/threonine-protein kinase that has a critical role in mitosis. It is essential for the localization of a mitotic checkpoint protein, BUBR1 (22), which was suggested to be associated with STK15 and this interaction was thought having significant roles in the tumorigenesis and development of LSCC (23). A low expression of the BUB1 protein was found to be highly correlated with the tumorigenesis and progression of LSCC and was proposed as a biomarker for the prognosis of LSCC (24). The present results indicated that $B U B 1$ was markedly enriched in the cell cycle pathways, consistent with previous functional enrichment analysis in LSCC (25). Additionally, BUB1 was prominent in the co-expressed network, suggesting this gene has a significant role in the development of LSCC.

TTK is another mitotic checkpoint protein that is correlated with cell proliferation and is responsible for the accurate segregation of chromosomes during mitosis (26). Dysregulation of this gene was verified to be associated with human cancers such as bladder and ovarian cancer (27). Considering that this gene was a vital node in the co-expressed network and also involved in the cell cycle pathway in the present study, it may play a significant role in the progression of LSCC from the early to the advanced stage via participation of the cell cycle-mediated pathway.

The TF E2F1 belongs to the E2F family, which plays a critical role in the control of cell cycle and the action of tumor suppressor. A p53 independent target of E2F1, the death inducing protein KIAA0767 was differentially expressed in human lung SCC (27). Additionally, the overexpression of $U H R F 1$, another target of $\mathrm{E} 2 \mathrm{~F} 1$, which played a pivotal function in promoting G1/S transition, was involved in the progression of LSCC and thus was considered as a useful biomarker for the detection of LSCC (29). Thus, the targets of E2F1 may contribute to the progression of LSCC. Combining our results that E2F1 was exhibited as a vital node in the co-expressed network, which was significantly enriched in the cell cycle pathway and predicted as a TSG, it may be hypothesized that the E2F1-mediated target expression may affect cell cycle and thus account for the progression of LSCC. However, more specific targets of the E2F1 should be established.

CEP55 is known to have a significant role in mitotic exit and cytokinesis. During interphase, CEP55 is localized to the mother centriole and the phosphorylation of this protein was required for the recruitment to midbody and cytokinesis (30). The oncogene FOXM1 (forkhead box MI) encoded protein is a transcriptional activator involved in cell proliferation. The evidence that two downstream targets of FOXM1, CEP55 and HELLS were positively associated with pre-malignancy and HNSCC development conferred the possibility that the two genes may be used as a biomarker in conjunction with FOXM1 for the early detection of LSCC (31). Of note, our results predicted CEP55 as an oncogene based on the information of 
TAG database, suggesting that CEP55 may exert its role in the development of LSCC as an oncogene.

Proteins encoded by homeobox genes are highly conserved TFs that have vital roles in morphogenesis in multicellular organisms. There are four similar homeobox gene clusters including $H O X A, H O X B, H O X C$ and $H O X D$ in mammals. It was found that $H O X 9$ was highly expressed in LSCC and acted as an oncogene (32). HOTAIR is a long intergenic non-coding RNA, which is transcribed by HOX locus. Overexpression of HOTAIR was observed in primary LSCC and associated with advanced stage of LSCC and suggested as a novel biomarker in the treatment of LSCC (4). The aforementioned findings indicate that the HOX family has a significant role in the regulation of LSCC. Notably, HOX genes were identified as TFs in the present results. Although no relevant evidence was provided for the role of HOX genes as TFs, it was suggested that these genes are important in the progression of LSCC.

The proteolytic activity of MMPs against extracellular matrix confers the significant role of MMPs in the invasion and metastasis in tumors, which contributes to the progression into the advanced stage. The immunohistochemical experiment demonstrated the correlation of MMP1 and TIMP1 (a tissue inhibitor of MMPs), which was important in the progression of LSCC (33). Additionally, the MMP9 expression was significantly associated with the metastasis and prognosis of LSCC and was suggested as an indicator for lymph node metastasis and tumor invasion in $\operatorname{LSCC}(34,35)$. A further study corroborated that overexpression of the MMP9 protein is a characteristic in LSCC and closely correlated with the increase of the tumor-node-metastasis stage (36), consistent with our present observation that $M M P 9$ was a tumor stagedependent increasing DEG, suggesting the critical role of $M M P 9$ in the development of LSCC.

$M M P 3$ is also associated with metastatic progression. The upregulation of $M M P 3$ was validated and identified as a signature in oral squamous cell carcinoma progression (37). Immunohistochemistry revealed the involvement of $M M P 3$ in the events correlated with lip SCC (38). Although not validated, $M M P 3$ was found to be upregulated in LSCC and associated with the development of invasion (39). Notably, MMP3 was confirmed to activate $M M P 1$ and the high expression of $M M P 1$ and $M M P 3$ was connected with tumor invasion and metastasis in LSCC (40). Our PPI network indicated the relationships between MMP1and MMP3, which gave potent evidence that regulations may exist between the two MMPs in the progression of LSCC.

The $A D H$ genes encode the alcohol dehydrogenase family protein. There was substantial evidence that $A D H$ genes (such as $A D H 1 B, A D H 1 C$ and $A D H 7$ ) were involved in the risk of various types of cancer including gastric and upper aero-digestive cancers $(41,42)$. With regard to their correlation with LSCC, a meta-analysis demonstrated that the polymorphisms of alcohol dehydrogenase genes $(A D H 1 B$ and $A D H 1 C$ ) were closely associated with head and neck cancer risk (43) . In the present study, four $A D H$ genes such as $A D H 1 A, A D H 1 C, A D H 4$ and $\mathrm{ADH} 7$ were downregulated in the two samples and showed a tumor stage-dependent decreasing pattern, suggesting that these genes could be used as signatures in the progression of LSCC.

$P L A U$ encodes a serine protease responsible for the extracellular matrix degradation and tumor cell migration and proliferation (44), which may account for its positive tumor stage being correlated with the expression of LSCC, as revealed in our study. In breast cancer cells, PLAU was indicated to have a marked role in transformation by affecting invasiveness (45). Gene amplification has been extensively demonstrated to contribute to tumor cell growth. In prostatic cancer (PC), it was verified that PLAU amplification was preferentially found in the advanced stage, but not detected in the benign prostatic hyperplasia (46), suggesting that this gene may have a tumor stage-dependent expression pattern. Notably, $M M P 3$ and $P L A U$ were involved in the plasminogen activator pathway and induced by $E R G$ (v-ets avian erythroblastosis virus E26 oncogene homolog) in PC (47). Given that MMP and PLAU were directly connected in our PPI network, it was hypothesized that a potential regulation may exist between these genes in the development of LSCC.

In conclusion, a set of critical genes in the progression of LSCC were identified including BUB1, TTK, E2F1, CEP55, $M M P s$ (MMPI, MMP9 and MMP3), PLAU, HOX genes and $A D H$ genes. Of these, MMPs, PLAU and $A D H$ genes were used as tumor stage-dependent biomarkers for the prognosis of LSCC. During the progression of LSCC, BUB1, TTK, E2F1 and CEP55 may exert their roles via the involvement of the cell cycle pathway and $H O X$ genes may serve as TFs, while $M M P 3$ may be mediated by MMP1. Additionally, $A D H$ genes may be used as indicators of the prognosis of LSCC. However, the findings remain to be verified.

\section{Acknowledgements}

The present study was supported by a grant from the Natural Science Foundation of Liaoning Province (no. 201202287).

\section{References}

1. Hui AB, Lenarduzzi M, Krushel T, Waldron L, Pintilie M, Shi W, Perez-Ordonez B, Jurisica I, O'Sullivan B, Waldron J, et al: Comprehensive MicroRNA profiling for head and neck squamous cell carcinomas. Clin Cancer Res 16: 1129-1139, 2010.

2. Wang W, Lin P, Han C, Cai W, Zhao X and Sun B: Vasculogenic mimicry contributes to lymph node metastasis of laryngeal squamous cell carcinoma. J Exp Clin Cancer Res 29: 60, 2010.

3. Zhang S-Y, Lu Z-M, Luo X-N, Chen LS, Ge PJ, Song XH, Chen SH and Wu YL: Retrospective analysis of prognostic factors in 205 patients with laryngeal squamous cell carcinoma who underwent surgical treatment. PLoS One 8: e60157, 2013.

4. Li D, Feng J, Wu T, Wang Y, Sun Y, Ren J and Liu M: Long intergenic noncoding RNA HOTAIR is overexpressed and regulates PTEN methylation in laryngeal squamous cell carcinoma. Am J Pathol 182: 64-70, 2013.

5. Karatas OF, Yuceturk B, Suer I, Yilmaz M, Cansiz H, Solak M, Ittmann $\mathrm{M}$ and Ozen $\mathrm{M}$ : The role of miR-145 in Human Laryngeal Squamous Cell Carcinoma. Head Neck n/a, 2014.

6. Rudolph E, Dyckhoff G, Becher H, Dietz A and Ramroth H: Effects of tumour stage, comorbidity and therapy on survival of laryngeal cancer patients: A systematic review and a meta-analysis. Eur Arch Otorhinolaryngol 268: 165-179, 2011.

7. Liu WW,Zeng ZY, Wu QL, Hou JH and Chen YY: Overexpression of MMP-2 in laryngeal squamous cell carcinoma: A potential indicator for poor prognosis. Otolaryngol Head Neck Surg 132: 395-400, 2005

8. Shi Y, Gong H-L, Zhou L, Tian J and Wang Y: CD24: A novel cancer biomarker in laryngeal squamous cell carcinoma. ORL J Otorhinolaryngol Relat Spec 74: 78-85, 2012.

9. Chen K, Cornejo KM, Ye W, Wu Q, Liang J and Jiang Z: Oncofetal protein IMP3: A new diagnostic biomarker for laryngeal carcinoma. Hum Pathol 44: 2126-2131, 2013. 
10. Geomela P-A, Kontos CK, Yiotakis I, Fragoulis EG and Scorilas A: L-DOPA decarboxylase mRNA expression is associated with tumor stage and size in head and neck squamous cell carcinoma: A retrospective cohort study. BMC Cancer 12: 484, 2012.

11. Saglam O, Shah V and Worsham MJ: Molecular differentiation of early and late stage laryngeal squamous cell carcinoma: An exploratory analysis. Diagn Mol Pathol 16: 218-221, 2007.

12. Plaça JR, Pinheiro DG, Panepucci RA, et al: Gene expression analysis of laryngeal squamous cell carcinoma. Genomics Data 5: 9-12, 2015.

13. Smyth GK: Limma: Linear models for microarray data. In: Bioinformatics and Computational Biology Solutions using $\mathrm{R}$ and Bioconductor. Springer, New York, NY, pp397-420, 2005.

14. Blake JA, Dolan M, Drabkin H, Hill DP, Li N, Sitnikov D, Bridges S, Burgess S, Buza T, McCarthy F, et al; Gene Ontology Consortium: Gene Ontology annotations and resources. Nucleic Acids Res 41 (D1): D530-D535, 2013.

15. Kanehisa M, Goto S, Sato Y, Furumichi $M$ and Tanabe $M$ KEGG for integration and interpretation of large-scale molecular data sets. Nucleic Acids Res 40: D109-D114, 2012.

16. Dennis G Jr, Sherman BT, Hosack DA, Yang J, Gao W, Lane HC and Lempicki RA: DAVID: Database for annotation, visualization, and integrated discovery. Genome Biol 4: 3, 2003.

17. Matys V, Kel-Margoulis OV, Fricke E, Liebich I, Land S, Barre-Dirrie A, Reuter I, Chekmenev D, Krull M, Hornischer K, et al: TRANSFAC and its module TRANSCompel: Transcriptional gene regulation in eukaryotes. Nucleic Acids Res 34: D108-D110, 2006.

18. Zhao M, Sun J and Zhao Z: TSGene: A web resource for tumor suppressor genes. Nucleic Acids Res 41: D970-D976, 2013.

19. Szklarczyk D, Franceschini A, Kuhn M, Simonovic M, Roth A, Minguez P, Doerks T, Stark M, Muller J, Bork P, et al: The STRING database in 2011: Functional interaction networks of proteins, globally integrated and scored. Nucleic Acids Res 39: D561-D568, 2011.

20. Kohl M, Wiese S and Warscheid B: Cytoscape: Software for Visualization and Analysis of Biological Networks. In: Data Mining in Proteomics. Springer, pp291-303, 2011.

21. Mattingly C, Rosenstein M, Colby G, Forrest J Jr and Boyer J: The Comparative Toxicogenomics Database (CTD): A resource for comparative toxicological studies. J Exp Zool A Comp. Exp Biol 305: 689-692, 2006.

22. Johnson VL, Scott MI, Holt SV, Hussein D and Taylor SS: Bub1 is required for kinetochore localization of BubR1, Cenp-E Cenp-F and Mad2, and chromosome congression. J Cell Sci 117: $1577-1589,2004$

23. Ma HF, Guo X and Li XY: Expression and Interaction between STK15 and BUBR1 in Human Laryngeal Squamous Cell Carcinoma. J China Med Univ 7: 017, 2011.

24. Li Z, Chen Y, Yan F, Zhang Q, Zhou J and Yang J: [The expression of BUB1 in laryngeal squamous cell carcinoma and its clinical significance]. Lin Chung Er Bi Yan Hou Tou Jing Wai Ke Za Zhi 27: 1184-1187, 2013 (In Chinese)

25. Liu Y, Sun W, Zhang K, Zheng H, Ma Y, Lin D, Zhang X, Feng L, Lei $\mathrm{W}$, Zhang Z, et al: Identification of genes differentially expressed in human primary lung squamous cell carcinoma. Lung Cancer 56: 307-317, 2007.

26. Dou Z, Ding X, Zereshki A, Zhang Y, Zhang J, Wang F, Sun J, Huang $\mathrm{H}$ and Yao X: TTK kinase is essential for the centrosomal localization of TACC2. FEBS Lett 572: 51-56, 2004

27. Ahmed AA, Lu Z, Jennings NB, Etemadmoghadam D, Capalbo L, Jacamo RO, Barbosa-Morais N, Le XF, Vivas-Mejia P, Lopez-Berestein G, et al; Australian Ovarian Cancer Study Group: SIK2 is a centrosome kinase required for bipolar mitotic spindle formation that provides a potential target for therapy in ovarian cancer. Cancer Cell 18: 109-121, 2010.

28. Sen S, Ateeq B, Sharma H, Datta P, Gupta SD, Bal S, Kumar A and Singh N: Identification of differentially expressed genes in human lung squamous cell carcinoma in Asian Indians using suppression subtractive hybridization. Clin Cancer Res 12 A71-A71, 2006.

29. Pi J-T, Lin Y, Quan Q, Chen LL, Jiang LZ, Chi W and Chen HY: Overexpression of UHRF1 is significantly associated with poor prognosis in laryngeal squamous cell carcinoma. Med Oncol 30: $613,2013$.
30. Fabbro M,Zhou B-B, Takahashi M, et al: Cdk1/Erk2- and Plk1dependent phosphorylation of a centrosome protein, Cep55, is required for its recruitment to midbody and cytokinesis. Dev Cell 9: 477-488, 2005

31. Waseem A, Ali M, Odell EW, Fortune F and Teh MT: Downstream targets of FOXM1: CEP55 and HELLS are cancer progression markers of head and neck squamous cell carcinoma. Oral Oncol 46: 536-542, 2010.

32. Sun $X$, Liu B, Ji W, Ma X, Wang $X$ and Gu H: The role of HOXA9 in human laryngeal squamous cell carcinoma. Oncol Res 20: 467-472, 2013

33. Krecicki T, Fraczek M, Jelen M, Podhorska M, Szkudlarek T and Zatonski T: Expression of collagenase-1 (MMP-1), collagenase-3 (MMP-13) and tissue inhibitor of matrix metalloproteinase-1 (TIMP-1) in laryngeal squamous cell carcinomas. Eur Arch Otorhinolaryngol 260: 494-497, 2003.

34. Cao X-L, Xu R-J, Zheng YY, Liu J, Teng YS, Li Y and Zhu J: Expression of type IV collagen, metalloproteinase-2, metalloproteinase- 9 and tissue inhibitor of metalloproteinase-1 in laryngeal squamous cell carcinomas. Asian Pac J Cancer Prev 12: 3245-3249, 2011.

35. Qin G, Li W, Sun X, Zhu L and Chen Z: [Expression and significance of CD44v6 and MMP-9 in laryngeal squamous cell carcinoma]. Lin Chuang Er Bi Yan Hou Ke Za Zhi 19: 688-691, 2005.

36. Papadas TA, Naxakis SS, Mastronikolis NS, Stathas T, Karabekos NCh and Tsiambas E: Determination of matrix metalloproteinase 9 (MMP-9) protein expression in laryngeal squamous cell carcinomas based on digital image analysis. J BUON 18: 977-981, 2013.

37. Nagaraj NS: Evolving 'omics' technologies for diagnostics of head and neck cancer. Brief Funct Genomics 8: 49-59, 2009.

38. Xavier FCA, Rodini CO, Ramalho LMP, Mantesso A and Nunes FD: WNT-5A, but not matrix metalloproteinase 3 or $\beta$-catenin protein, expression is related to early stages of lip carcinogenesis. J Oral Pathol Med 38: 708-715, 2009.

39. Bogner W, Gruber S, Pinker K, Grabner G, Stadlbauer A, Weber M, Moser E, Helbich TH and Trattnig S: Diffusion-weighted MR for differentiation of breast lesions at 3.0 T: How does selection of diffusion protocols affect diagnosis? Radiology 253: 341-351, 2009.

40. Vachani A, Nebozhyn M, Singhal S, Alila L, Wakeam E, Muschel R, Powell CA, Gaffney P, Singh B, Brose MS, et al: A 10-gene classifier for distinguishing head and neck squamous cell carcinoma and lung squamous cell carcinoma. Clin Cancer Res 13: 2905-2915, 2007.

41. Hashibe M, McKay JD, Curado MP, Oliveira JC, Koifman S, Koifman R, Zaridze D, Shangina O, Wünsch-Filho V, Eluf-Neto J, et al: Multiple ADH genes are associated with upper aerodigestive cancers. Nat Genet 40: 707-709, 2008.

42. Duell EJ, Sala N, Travier N, Muñoz X, Boutron-Ruault MC, Clavel-Chapelon F, Barricarte A, Arriola L, Navarro C, SánchezCantalejo E, et al: Genetic variation in alcohol dehydrogenase (ADH1A, ADH1B, ADH1C, ADH7) and aldehyde dehydrogenase (ALDH2), alcohol consumption and gastric cancer risk in the European Prospective Investigation into Cancer and Nutrition (EPIC) cohort. Carcinogenesis 33: 361-367, 2012.

43. Chang JS, Straif K and Guha N: The role of alcohol dehydrogenase genes in head and neck cancers: A systematic review and meta-analysis of ADH1B and ADH1C. Mutagenesis 27: 275-286, 2012.

44. Jiang J, Loganathan J, Eliaz I, Terry C, Sandusky GE and Sliva D: ProstaCaid inhibits tumor growth in a xenograft model of human prostate cancer. Int J Oncol 40: 1339-1344, 2012.

45. Iliopoulos D, Rotem A and Struhl K: Inhibition of miR-193a expression by Max and RXR $\alpha$ activates K-Ras and PLAU to mediate distinct aspects of cellular transformation. Cancer Res 71: 5144-5153, 2011

46. Bloch M, Ousingsawat J, Simon R, Schraml P, Gasser TC, Mihatsch MJ, Kunzelmann K and Bubendorf L: KCNMA1 gene amplification promotes tumor cell proliferation in human prostate cancer. Oncogene 26: 2525-2534, 2007.

47. Tomlins SA, Laxman B, Varambally S, Cao X, Yu J, Helgeson BE, Cao Q, Prensner JR, Rubin MA, Shah RB, et al: Role of the TMPRSS2-ERG gene fusion in prostate cancer. Neoplasia 10: 177-188, 2008. 